\title{
Fast and Stable Contour Integration for High Order Divided Differences via Elliptic Functions
}

\author{
M. Lopez-Fernandez ${ }^{*} \quad$ S. Sauter ${ }^{\dagger}$
}

August 2, 2012

\begin{abstract}
In this paper, we will present a new method for evaluating high order divided differences for certain classes of analytic, possibly, operator values functions. This is a classical problem in numerical mathematics but also arises in new applications such as, e.g., the use of generalized convolution quadrature to solve retarded potential integral equations. The functions which we will consider are allowed to grow exponentially to the left complex half plane and the interpolation points are scattered in a large real interval. Our approach is based on the representation of divided differences as contour integral and we will employ a subtle parameterization of the contour in combination with a quadrature approximation by the trapezoidal rule.
\end{abstract}

Keywords: Divided Differences, Numerical Approximation of Contour Integrals, Jacobi Elliptic Functions, Convolution Quadrature.

Mathematics Subject Classification (2000): 30E20, 33B99, 39A70, 65D30, 65R20

\section{Introduction}

In this paper, we consider the stable and efficient evaluation of high order divided differences via contour integrals. The interpolation points $x_{i}, 1 \leq i \leq N$, for the divided differences of a function $f$ are assumed to be positive and contained in an interval $\mathcal{I}=[m, M]$ with $0<m<M$. The function $f$ is assumed to be analytic in a half plane

$$
\mathbb{C}_{\sigma}:=\{z \in \mathbb{C} \mid \operatorname{Re} z>\sigma\}
$$

\footnotetext{
*Institut für Mathematik, Universität Zürich, Winterthurerstrasse 190, CH-8057 Zürich, Switzerland, e-mail: maria.lopez@math.uzh.ch

${ }^{\dagger}$ Institut für Mathematik, Universität Zürich, Winterthurerstrasse 190, CH-8057 Zürich, Switzerland, e-mail: stas@math.uzh.ch
} 
for some $\sigma<-1$. We allow exponential growth to the left complex half plane and polynomial growth to the right half plane. The problem of evaluating high order divided differences or high order derivatives for such type of functions arise, e.g., when the convolution quadrature method [17], [18], [12], [4], [3] with variable step size [15] is employed to approximate convolution operators, in particular, for hyperbolic differential equations [10], [2], [17].

Another application is the expansion of a function into an interpolating polynomials via Newton's divided differences. For a real sequence $x_{i}, 1 \leq i \leq$ $N+1$, of interpolation points with given function values $f_{i}, 1 \leq i \leq N+1$ the interpolating polynomial of degree $N$ can be written in the form

$$
\sum_{i=1}^{N+1}\left(\left[x_{1}, x_{2}, \ldots, x_{i}\right] f\right) \omega_{1, i-1}
$$

with the Newton polynomials

$$
\omega_{1, i}(x)=\prod_{k=1}^{i}\left(x-x_{k}\right) .
$$

Its evaluation at some point $x$ requires the accurate and efficient evaluation of the product $\left(\left[x_{1}, x_{2}, \ldots, x_{i}\right] f\right) \omega_{1, i-1}(x)$. However, the evaluation of high order divided differences by Newton's table becomes very unstable if $N$ becomes large and/or the ratio $M / m$ increases. Furthermore, the interpolation points $x_{i}$, possibly, are generated recursively, e.g., by an adaptive time stepping scheme, and their multiplicity can be high and varying. In the latter case, significant parts of Newton's table for computing divided differences have to be recomputed in each step for new interpolation points. An additional difficulty arises if the distances between the interpolation points are very small causing again severe roundoff problems.

As an alternative to Newton's table for computing divided differences we will employ here their representation as contour integrals and a discretization by the trapezoidal rule. However, the choice of the contour is very delicate if the ratio $M / m$ is large. As our contour, we choose here a circle about $M$ with radius $M$. However, the simple parametrization by $M(1+\exp (2 \pi \alpha))$ results in a very slow convergence if the ratio $M / m$ becomes large. A much better behavior is obtained by using a translation and dilation of a composition of a Jacobi elliptic function with a Möbius transform - this idea was used in [13] to compute matrix functions via contour integrals. Our numerical experiments will show that this choice leads to an exponentially convergent approximation. The theory will be based on classical quadrature estimates for the trapezoidal rule for periodic functions which can be extended analytically to some complex strip around the integration interval. Since a Jacobi elliptic function is involved the error analysis requires some technical estimates of this function. The choice of our integration contour ensures that severe problems with roundoff errors which often arise when integrating highly oscillatory functions are avoided.

The evaluation of divided differences is a classical problem in numerical analysis. However the accurate evaluation of high order divided differences for quite 
general and, possibly, adaptively selected nodal points with changing multiplicities is still a challenging problem. For the evaluation of divided differences for the exponential function this is well known; in [19] an improved procedure is presented which combines the traditional recurrence with special properties of the exponential function. This method has been further developed in [5] for the matrix exponential propagator, while fast versions of the traditional recurrence are presented, e.g., in [21] an [9]. However, as explained above, our applications $[15,16]$ involve more general functions and quite general, possibly, highly nonuniform and adaptively selected nodal points with varying multiplicities and do not fit into the classes of functions which are considered in these references.

The paper is organized as follows. In Section 2 we will present the new method for computing divided differences via contour integrals and the particular choice of the contour. Section 3 is devoted to the error analysis for the approximation of contour integrals by the trapezoidal rule and will be based on analytic function theory. In Section 4 we will analyze the width of the complex neighborhood of the contour where the function $f$ can be extended analytically. This involves some technical estimates for Jacobi elliptic functions. Finally, in Section 5 we will present some numerical experiments which show the efficiency of our approximation method.

\section{Divided Differences via Contour Integrals}

Let a finite sequence of positive points $\left(x_{i}\right)_{i=1}^{N}$ be given which satisfies ${ }^{1}$

$$
1 \leq m:=\min _{1 \leq i \leq N} x_{i}
$$

For fixed $c_{0} \in(0,1)$, we choose

$$
M \geq \max \left\{\max _{1 \leq i \leq N} x_{i}, \frac{1}{1-c_{0}} m\right\}
$$

such that the inverval $\mathcal{I}:=[m, M]$ contains all points $x_{i}$ and the quotient $q:=M / m$ satisfies

$$
q-1 \geq c_{0} q
$$

Remark 1 Our quadrature error estimates typically will contain a factor $q /(q-1)$ which is large if $M$ is chosen very close to $m$, i.e., if $q$ is close to 1 . To circumvent this theoretical artifact, we have therefore taken the freedom to choose $M$ large enough such that all nodal points are contained in $\mathcal{I}$ and $q /(q-1)$ is bounded from above by the fixed constant $c_{0}^{-1}$. We emphasize that this choice obviously covers also the case that all nodal points are equal since the choice $M=m /\left(1-c_{0}\right)$ is then admissible.

\footnotetext{
${ }^{1}$ We have chosen here the condition $m \geq 1$ instead of $m>0$ to reduce technicalities.
} 
For a function $f: \mathcal{I} \rightarrow V$ with values in some normed linear space $V$ we denote by

$$
\delta_{1, N}(f):=\left[x_{1}, x_{2}, \ldots, x_{N}\right] f
$$

the divided difference of $f$ with respect to these points with standard modifications for points with multiplicities larger than 1 (see, e.g., [22]). Our goal is to present a fast and stable method to evaluate $\delta_{1, N}(f)$ for a certain class of functions. As outlined in the introduction, the direct evaluation of (high order) divided differences becomes very unstable because of roundoff effects if the distances between the mesh points $x_{i}$ become very small or $N$ becomes large. In addition, the points $x_{i}$ might be generated recursively so that they are, typically, not ordered and they change their multiplicities. This requires some recursive reordering of the Newton table for computing divided differences and the new computation of substantial parts of the Newton table for a newly generated point $x_{N}$.

Definition 2 For $\sigma \in \mathbb{R}, p \in \mathbb{R}, \alpha>0, \beta \geq 0$, the set $\mathcal{A}(\sigma, p, \alpha, \beta)$ contains all functions $f: \mathbb{C}_{\sigma} \rightarrow V$ that satisfy

1. $f$ is analytic in $\mathbb{C}_{\sigma}$

2. $f$ satisfies the growth estimate

$$
|f(z)| \leq \alpha\left(1+\mathrm{e}^{-\beta \operatorname{Re} z}\right)(\max \{1,|z|\})^{p} \quad \forall z \in \mathbb{C}_{\sigma} .
$$

For the set of points $\left(x_{i}\right)_{i=1}^{N}$, the Newton polynomial is defined (cf. (1)) by

$$
\omega_{1, N}(z)=\prod_{i=1}^{N}\left(z-x_{i}\right) .
$$

From $[22$, p. 54] we conclude that the divided difference can be written in the form

$$
\delta_{1, N}(f)=\frac{1}{2 \pi \mathrm{i}} \int_{\mathcal{C}} \frac{f(z)}{\omega_{1, N}(z)} d z,
$$

where $\mathcal{C}$ is any closed contour in $\mathbb{C}_{\sigma}$ which contains $\mathcal{I}$ and is oriented counterclockwise. Our approach for approximating divided differences is based on the approximation of the contour integral by a trapezoidal rule.

Remark 3 The choice of the contour and its parametrization is a very delicate problem if the quotient $q=M / m$ becomes large. If $\mathcal{C}$ is "too" close to the interval $\mathcal{I}$ then the denominator in (4) becomes very small and numerical quadrature will suffer. If $\mathcal{C}$ is "too" far from $\mathcal{I}$ - in particular if $\mathcal{C}$ enters the left half of the complex plane, the exponential growth of the term $\mathrm{e}^{-\beta \operatorname{Re} z}$ for $\operatorname{Re} z \ll 0$ will spoil the quadrature accuracy. 
As a compromise we have chosen $\mathcal{C}$ as the $\operatorname{circle} \mathcal{C}_{M}$ about $M$ with radius $M$. For an interval $J=\overline{A B} \subset \mathbb{C}$ with length $|J|:=|B-A|$, let $\gamma_{M}: J \rightarrow \mathcal{C}_{M}$ denote a $|J|$-periodic parametrization of $\mathcal{C}_{M}$ which can be extended analytically in a complex neighborhood of $J$. Then the approximation of the divided difference is given by applying the trapezoidal rule for periodic functions to the parametrized integral

$$
\begin{aligned}
\delta_{1, N}(f) & =\frac{1}{2 \pi \mathrm{i}} \int_{J} \frac{f \circ \gamma_{M}(\sigma)}{\omega_{1, N}\left(\gamma_{M}(\sigma)\right)} \gamma_{M}^{\prime}(\sigma) d \sigma \\
& \approx \frac{|J|}{2 \pi \mathrm{i} N_{Q}} \sum_{\ell=0}^{N_{Q}-1} \frac{f \circ \gamma_{M}\left(t_{\ell}\right)}{\omega_{1, N}\left(\gamma_{M}\left(t_{\ell}\right)\right)} \gamma_{M}^{\prime}\left(t_{\ell}\right)=: \tilde{\delta}_{1, N}(f),
\end{aligned}
$$

where $t_{\ell}=A+\frac{\ell}{N_{Q}}(B-A)$.

Next, we will introduce the concrete choice of the parametrization $\gamma_{M}$ being motivated by the quadrature error analysis for analytic periodic functions. For the sake of simplicity we assume that $\operatorname{Im} A=\operatorname{Im} B$ and, for $\rho>0$, we define the extension of the parameter interval $J=\overline{A B}$ to a complex horizontal strip

$$
\mathfrak{s}_{\rho}(J):=\{t+\mathrm{i} v: t \in J \wedge-\rho \leq v \leq \rho\} .
$$

We assume that $\gamma_{M}$ can be extended analytically to $\mathfrak{s}_{\rho}(J)$ for some $\rho>0$ and we define the mapped region under $\gamma_{M}$ by

$$
\mathcal{C}_{M, \rho}:=\left\{\gamma_{M}(\sigma): \sigma \in \mathfrak{s}_{\rho}(J)\right\} .
$$

An important quantity in the error estimate is the modulus

$$
\mathfrak{M}_{M, \rho}(f):=\sup _{z \in \mathcal{C}_{M, \rho}}\left|\frac{f(z)}{\omega_{1, N}(z)}\right| .
$$

Since we allow that $f$ grows exponentially with increasing negative real part of $z$ the parametrization $\gamma_{M}$ should be chosen such that

$$
\mu(M, \rho):=\min \left\{\operatorname{Re} z: z \in \mathcal{C}_{M, \rho}\right\}
$$

grows "relatively" slowly to the negative half plane as a function of $\rho$. Note that the standard parametrization of $\mathcal{C}_{M}$ by $\gamma(t):=M\left(1+\mathrm{e}^{2 \pi \mathrm{i} t}\right), t \in[0,1]$ leads to a prohibitive large number of quadrature points if the ratio $M / m$ is large. This is due to the restricted width $\rho$ of the analyticity strip $\mathfrak{s}_{\rho}(J)$, which turns out to be limited by $m / M$, and to the "too" fast growth of $\mu(M, \rho)$ to the left half of the complex plane as $\rho$ increases.

A much better behavior is obtained by using a translation and dilation of a composition of a Jacobi elliptic function with a Möbius transform - this idea was used in [13] to compute matrix functions via contour integrals. The definition needs some preparatory steps. 
For any parameter $\lambda \in[0,1]$, let $\operatorname{pr}(\cdot \mid \lambda)$ denote the Jacobi elliptic function according to the definition in [1, Section 16.1], where $\mathrm{p}, \mathrm{r}$ is any two of the letters s, c, d, n. We write short

$$
\operatorname{sn}(\sigma)=\operatorname{sn}(\sigma \mid \lambda), \quad \operatorname{cn}(\sigma)=\operatorname{cn}(\sigma \mid \lambda), \quad \operatorname{dn}(\sigma)=\operatorname{dn}(\sigma \mid \lambda) .
$$

The complete elliptic integrals of the first kind are defined by

$$
\begin{aligned}
K(\lambda) & :=\int_{0}^{1} \frac{d x}{\sqrt{\left(1-x^{2}\right)\left(1-\lambda x^{2}\right)}} \quad(\text { see }[11,8.112(1 .) \text { and }(2 .)]) \\
K^{\prime}(\lambda) & :=K(1-\lambda) \quad(\text { see }[11,8.112(3 .) \text { and } 8.111(2 .)]) .
\end{aligned}
$$

Remark 4 Let $0<m<M<\infty$ and $q=\frac{M}{m} \in(1, \infty)$. We will consider $\lambda$ as a function of $q$, more precisely,

$$
\lambda=k^{2} \quad \text { with } \quad k=k(q)=\frac{q-\sqrt{2 q-1}}{q+\sqrt{2 q-1}} .
$$

Note that $k$ is strictly monotonously increasing and $0=k(1) \leq k(q) \leq k(\infty)=$ 1.

For later use we note that the choice of $\lambda=k^{2}(q)$ allows the following estimate

$$
\frac{1}{\sqrt{q}} \leq\left|1-\lambda^{1 / 2}\right| \leq 2 \sqrt{\frac{2}{q}} \text { and } \frac{1}{2 \sqrt{q}} \leq\left|1-\lambda^{1 / 4}\right| \leq 2 \sqrt{\frac{2}{q}} .
$$

We introduce the parameter interval

$$
J_{\lambda}:=\overline{P_{\lambda} Q_{\lambda}} \quad \text { with } P_{\lambda}=-K(\lambda)+\frac{\mathrm{i}}{2} K^{\prime}(\lambda) \text { and } Q_{\lambda}=3 K(\lambda)+\frac{\mathrm{i}}{2} K^{\prime}(\lambda) .
$$

Definition 5 Let $\lambda$ and $k$ be the functions of $q=M / m$ as in (11). The parametrization of the integration contour $\mathcal{C}_{M}$ is given by $\gamma_{M}(\sigma)=(z \circ u)(\sigma)$, where

$$
z(u):=\frac{M}{q-1}\left(\sqrt{2 q-1} \frac{\lambda^{-1 / 2}+u}{\lambda^{-1 / 2}-u}-1\right), \quad u(\sigma):=\operatorname{sn}(\sigma, \lambda), \quad \sigma \in J_{\lambda} .
$$

Remark 6 The function $\gamma_{M}$ as in Definition 5 is a parametrization of the circle $\mathcal{C}_{M}$ (cf. Lemma 15). Note that the orientation of $\gamma_{M}$ is clockwise, which by (5) leads to an approximation of $-\delta_{1, N}$.

With this choice of contour our quadrature approximation reads

$$
\delta_{1, N}(f) \approx \sum_{\ell=0}^{N_{Q}-1} w_{\ell} \frac{f\left(z_{\ell}\right)}{\omega_{1, N}\left(z_{\ell}\right)},
$$

with

$$
z_{\ell}=\gamma_{M}\left(\sigma_{\ell}\right), \quad w_{\ell}=\frac{4 K(\lambda)}{2 \pi \mathrm{i} N_{Q}} \gamma_{M}^{\prime}\left(\sigma_{\ell}\right), \quad \text { and } \quad \sigma_{\ell}=-K(\lambda)+\ell \frac{4 K(\lambda)}{N_{Q}},
$$


for $\ell=1, \ldots, N_{Q}$, where

$$
\gamma_{M}^{\prime}(\sigma)=\frac{M \sqrt{2 q-1}}{q-1} \frac{2 \mathrm{cn}(\sigma) \operatorname{dn}(\sigma)}{k\left(k^{-1}-\operatorname{sn}(\sigma)\right)^{2}} .
$$

The evaluation of the Jacobi elliptic functions and the elliptic integrals at complex arguments can be performed very efficiently and accurately in MATLAB by means of Driscoll's Schwarz-Christoffel Toolbox [7, 8] which is freely available online. In particular the functions ellipkkp and ellipjc are needed to compute (16), cf. [13].

\section{Error Analysis}

The error analysis is based on classical estimates of the trapezoidal rule when applied to periodic functions which can be extended analytically to a certain strip around the integration interval.

Remark 7 The derivation of the estimates in this and the next section is rather technical. We emphasize that all constants are positive real numbers - independent of the function $f$ and the parameters $m, M, \alpha, \beta, \sigma, p, \lambda, q, \rho$. They are chosen to simplify the expressions which are involved in the statements and proofs. We have avoided to optimize their choice in order not to further increase technicalities and they should be regarded as a proof that "such constants exists".

In Figure 1 we show how the conformal transformation $\gamma_{M}$ maps parallel horizontal lines within the analyticity strip.

The following theorem estimates the width of the region $\mathcal{C}_{M, \rho}$.

Theorem 8 Let $c_{0} \in(0,1), m$, and $M$ be as in (2) and set $C_{0}:=\frac{370}{c_{0}}$. For any

$$
0 \leq \rho \leq\left(3 C_{0}\right)^{-1} \min \left\{M^{-1 / 2}, m^{-1}\right\}
$$

it holds

$$
\mu(M, \rho):=\min \left\{\operatorname{Re} z: z \in \mathcal{C}_{M, \rho}\right\} \geq-1
$$

and, for all $x \in \mathcal{I}$, we have

$$
\operatorname{dist}\left(x, \mathcal{C}_{M, \rho}\right) \geq\left(1-2 M^{-1 / 2}-\frac{2}{3} m^{-1}\right) x .
$$

For the modulus of $z \in \mathcal{C}_{M, \rho}$ it holds

$$
\max \left\{|z|: z \in \mathcal{C}_{M, \rho}\right\} \leq C_{1} M \quad \text { with } \quad C_{1}=10 / 3 .
$$

The proof of this theorem relies on some technical estimates of Jacobi's elliptic functions and will be postponed to Section 4 (cf. Theorem 20, Theorem 21, and Corollary 22).

We employ the following quadrature estimate for periodic functions. 

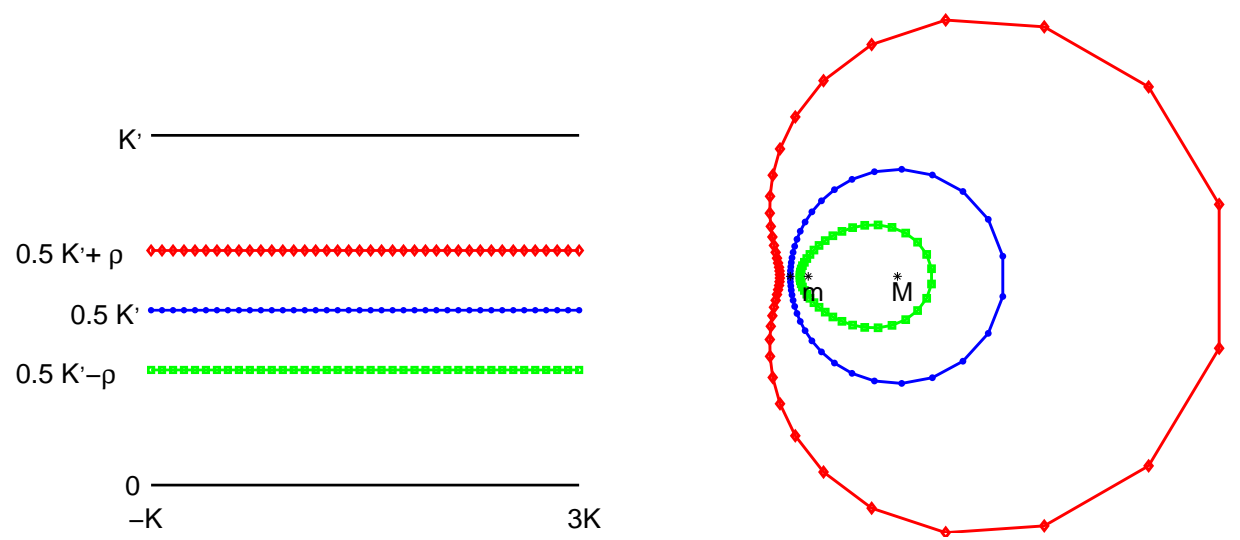

Figure 1: Illustration of the parametrization $\gamma_{M}$ in Definition 5. Left: Horizontal lines in the $\sigma$-domain. Right: The corresponding images in the $z$-domain. The outer contour is kidney-shaped while the inner one is egg-shaped.

Theorem 9 Let $J=\overline{A B} \subset \mathbb{C}$ and $\mathfrak{s}_{\rho}(J)$ be as in (6) and let $V$ be a normed linear space. For a periodic function $g: J \rightarrow V$ which can be extended to an analytic function in $\mathfrak{s}_{\rho}(J)$ for some $\rho>0$ the quadrature error

$$
E_{N_{Q}}(g):=\int_{J} g(\sigma) d \sigma-\frac{|J|}{N_{Q}} \sum_{\ell=0}^{N_{Q}-1} g\left(t_{\ell}\right) \quad \text { with } \quad t_{\ell}:=A+\frac{\ell}{N_{Q}}(B-A)
$$

can be estimated by

$$
\left\|E_{N_{Q}}(g)\right\|_{V} \leq|J| \frac{2}{\mathrm{e}^{\frac{2 \pi}{J J} N_{Q} \rho}-1} \sup _{\sigma \in \mathfrak{s}_{\rho}(J)}\|g(\sigma)\|_{V} .
$$

Proof. The proof goes back to [6] - concretely the estimate follows from [14, (28)] by employing the affine pullback

$$
\chi:[0,2 \pi] \rightarrow J, \quad \chi(t):=A+\frac{t}{2 \pi}(B-A) .
$$

Theorem 10 Let $c_{0} \in(m, M), 0<m<M$ be as in (2). Let $N$ be the number of points for the divided difference in (3) and let us define the ratio $R b y^{2}$

$$
R:=N\left(M^{-1 / 2}+m^{-1}\right) .
$$

\footnotetext{
${ }^{2}$ We can always assume $R \leq 2 N / m$ since we have the freedom (cf. (2b)) to choose $M \geq m^{2}$.
} 
Let $\lambda, k$ be the functions of $q=M / m$ as in (11) and let the function $f \in$ $\mathcal{A}(\sigma, p, \alpha, \beta)$ for some $\sigma<-1$. Then, for the divided difference approximation (5) via quadrature with the contour as in Definition 5 it holds

$$
\left|\omega_{1, N}(0)\right|\left\|\delta_{1, N}(f)-\tilde{\delta}_{1, N}(f)\right\|_{V} \leq \alpha \frac{C_{1}^{p} C_{2}}{\pi}(10+2 \log q) M^{p+1} \frac{\mathrm{e}^{2 \max (1, \beta) R}}{\mathrm{e}^{N_{Q} \tau}-1}
$$

with $\tau:=\frac{\pi}{3 C_{0}} \frac{\min \left\{M^{-1 / 2}, m^{-1}\right\}}{10+2 \log q}$.

Proof. In view of (5) we introduce the function $g: J_{\lambda} \rightarrow V$ by

$$
g(\sigma):=\frac{1}{2 \pi \mathrm{i}} \frac{f \circ \gamma_{M}(\sigma)}{\omega_{1, N}\left(\gamma_{M}(\sigma)\right)} \gamma_{M}^{\prime}(\sigma) .
$$

Let $\rho=\left(3 C_{0}\right)^{-1} \min \left\{M^{-1 / 2}, m^{-1}\right\}$ (cf. (18)). Then, Theorem 8 directly implies an estimate of $\mathfrak{M}_{M, \rho}(f)$ in (8). Note that (19a) yields

$$
\sup _{z \in \mathcal{C}_{M, \rho}}|f(z)| \leq \sup _{z \in \mathcal{C}_{M, \rho}} \alpha\left(1+\mathrm{e}^{-\beta \operatorname{Re} z}\right)(\max \{1,|z|\})^{p} \leq \alpha\left(C_{1} M\right)^{p}\left(1+\mathrm{e}^{\beta}\right) .
$$

To estimate the denominator in (8) we start with

$$
\inf _{z \in \mathcal{C}_{M, \rho}}\left|z-x_{i}\right| \stackrel{(19 \mathrm{~b})}{\geq}(1-\varepsilon) x_{i} \quad \text { with } \quad \varepsilon:=2\left(\frac{1}{\sqrt{M}}+\frac{1}{m}\right) .
$$

Thus,

$\sup _{z \in \mathcal{C}_{M, \rho}} \omega_{1, N}^{-1}(z) \leq\left|\omega_{1, N}^{-1}(0)\right|(1-\varepsilon)^{-N}=\left|\omega_{1, N}^{-1}(0)\right| \mathrm{e}^{\sum_{i=1}^{N} \log (1-\varepsilon)}=\left|\omega_{1, N}^{-1}(0)\right| \mathrm{e}^{2 R}$.

Hence,

$$
\left|\omega_{1, N}(0)\right| \mathfrak{M}_{M, \rho}(f) \leq \alpha\left(C_{1} M\right)^{p}\left(1+\mathrm{e}^{\beta}\right) \mathrm{e}^{2 R} .
$$

It remains to estimate $\gamma_{M}^{\prime}$ in the strip $\mathfrak{s}_{\rho}\left(J_{\lambda}\right)$. We use Corollary 17 below, the choice of $\rho$, and $q^{1 / 2} M^{-1 / 2} \leq 1$ (which follows from (2a)) to obtain for all $\sigma \in \mathfrak{s}_{\rho}\left(J_{\lambda}\right)$ the estimate

$$
\left|\gamma_{M}^{\prime}(\sigma)\right| \leq C_{2} M \quad \text { with } \quad C_{2}:=\frac{65}{c_{0}} \sqrt{2}
$$

Hence,

$$
\left|\omega_{1, N}(0)\right| \sup _{\sigma \in \mathfrak{s}_{\rho}(J)}\|g(\sigma)\|_{V} \leq \frac{C_{1}^{p} C_{2}}{2 \pi} \alpha M^{p+1}\left(1+\mathrm{e}^{\beta}\right) \mathrm{e}^{2 R} .
$$

To apply Theorem 9 we have to estimate the interval length $\left|J_{\lambda}\right|=4 K(\lambda)$ and employ Lemma 23 to obtain

$$
\begin{aligned}
\left|J_{\lambda}\right| & \leq \frac{1}{2} \log \frac{16}{1-\lambda}\left(1+\sqrt{\frac{2}{7}}(1-\lambda)\right) \stackrel{(12)}{\leq} \frac{1}{2} \log (16 \sqrt{q})\left(1+16 \sqrt{\frac{1}{7 q}}\right) \\
& \leq 10+2 \log q .
\end{aligned}
$$


Hence, we get

$$
\frac{\pi}{\left|J_{\lambda}\right|} \rho \geq \frac{\pi}{3 C_{0}} \frac{\min \left\{M^{-1 / 2}, m^{-1}\right\}}{10+2 \log q} .
$$

Corollary 11 Let the assumptions of Theorem 10 be satisfied and let $\tau$ and $R$ be as in Theorem 10. Then, the choice of the number of quadrature points according to

$$
N_{Q}>\tau^{-1}\left(c+\log \left(\frac{1}{\varepsilon}\right)+2 \max (1, \beta) R+(p+2) \log M\right)
$$

with $c \geq \log \left(2+20 \alpha \frac{C_{1}^{p} C_{2}}{\pi}\right)$ implies an accuracy $\varepsilon$ in the approximation (5) of the divided difference in (3), i.e.,

$$
\left|\omega_{1, N}(0)\right|\left\|\delta_{1, N}(f)-\tilde{\delta}_{1, N}(f)\right\|_{V} \leq \varepsilon .
$$

Proof. Since $N_{Q} \geq \tau^{-1} \log 2$, the denominator in (20) can be estimated from below by $\mathrm{e}^{N_{Q} \tau} / 2$ and we estimate the term $(10+2 \log q)$ in (20), generously, by $10 M$ (cf. Remark 7 ). In view of (20) we obtain

$$
\left|\omega_{1, N}(0)\right|\left\|\delta_{1, N}(f)-\tilde{\delta}_{1, N}(f)\right\|_{V} \leq 20 \alpha \frac{C_{1}^{p} C_{2}}{\pi} \mathrm{e}^{2 \max (1, \beta) R+(p+2) \log M-N_{Q} \tau} \stackrel{(21)}{\leq} \varepsilon .
$$

We finally present a version of Theorem 10 , where we assume that the mesh is graded at most quadratically, i.e.,

$$
\max _{1 \leq i \leq N} x_{i} \leq m^{2} .
$$

In this case, we may choose $M:=m^{2}$. In many applications we also have $N \leq m$ (generalizations to $M \leq C m^{2}$ and $N \leq C^{\prime} m$ are straightforward). This case is considered in the following Corollary.

Corollary 12 Let $N \leq m$ and assume that (22) is satisfied. Let the assumptions of Theorem 10 be satisfied. Let $\lambda, k$ be the functions of $q=M / m$ as in (11) and let $f \in \mathcal{A}(\sigma, p, \alpha, \beta)$ for some $\sigma<-1$. Then the choice of quadrature points according to

$$
N_{Q}>m \log (m+1)\left(c_{2}+c_{1} \log \left(\frac{1}{\varepsilon}\right)+c_{3} \log m\right)
$$

with some constants

$$
c_{1}:=\frac{30 C_{0}}{\pi \log 2}, \quad c_{2}:=c c_{1}, \quad c_{2} \geq 4 c_{1} \max (1, \beta)
$$

leads to

$$
\left|\omega_{1, N}(0)\right|\left\|\delta_{1, N}(f)-\tilde{\delta}_{1, N}(f)\right\|_{V} \leq \varepsilon
$$


Proof. The result follows from Corollary 11 by using the assumptions on $N$, $m$, and $N_{Q}$ in combination with

$$
\tau^{-1}=\frac{3 C_{0}}{\pi}(10+2 \log q) \max \left(M^{1 / 2}, m\right)=\frac{3 C_{0}}{\pi}(10+2 \log m) m \leq \frac{30 C_{0}}{\pi \log 2} m \log (m+1) .
$$

\section{Estimates of Jacobi Elliptic Functions}

In this section, we will prove Theorem 8. For this we have to analyze the behavior of the Jaocbi elliptic function in a neighborhood of the interval $J_{\lambda}$.

In order to estimate the distance from our integration contour to the boundary of the region $C_{M, \rho}$ (cf. (7)), we have to estimate the derivatives of $\gamma_{M}$ and, in turn, the derivatives of $z$ and $u$ (cf. (14)). Let $\mathbb{S}_{1}:=\{z \in \mathbb{C}|| z \mid=1\}$.

In order to give an idea of the behavior of the width of the region $\mathcal{C}_{M, \rho}$, we point out the following special values of $\gamma_{M}$ and $\gamma_{M}^{\prime}$ (cf. [1, Sec. 16.5])

$$
\begin{array}{ll}
\gamma_{M}\left(-K+\frac{\mathrm{i}}{2} K^{\prime}\right)=0, & \gamma_{M}^{\prime}\left(-K+\frac{\mathrm{i}}{2} K^{\prime}\right)=O(m) \\
\gamma_{M}\left(0+\frac{\mathrm{i}}{2} K^{\prime}\right)=m+\mathrm{i} m \sqrt{2 q-1}, & \gamma_{M}^{\prime}\left(-K+\frac{\mathrm{i}}{2} K^{\prime}\right)=O(\sqrt{M m}) \\
\gamma_{M}\left(K+\frac{\mathrm{i}}{2} K^{\prime}\right)=2 M, & \gamma_{M}^{\prime}\left(-K+\frac{\mathrm{i}}{2} K^{\prime}\right)=O(M)
\end{array}
$$

The above formulae indicate that the derivative of our parametrization gets smaller as our contour becomes closer to the imaginary axis. This property is essential in order to avoid that the boundary of the region $C_{M, \rho}$ enters "too" much the left half plane (where the integrand is allowed to grow exponentially) or getting too close to the poles in $\mathcal{I}$ (cf. Remark 3) as can be seen from the mean value theorem

$$
\gamma_{M}(\sigma+\rho \zeta)=\gamma_{M}(\sigma)+\rho \gamma_{M}^{\prime}(\xi)
$$

for some $\xi$ in between $\sigma$ and $\sigma+\rho \zeta$.

In the rest of this section we will derive sharp estimates for $\gamma_{M}^{\prime}$ and the width of $C_{M, \rho}$ which are explicit with respect to $\rho$ and all the parameters involved in the approximation formula (5). We have chosen the constants in the estimates such that the expressions becomes as simple as possible (cf. Remark 7) and, hence, avoided to optimize them.

Remark 13 For all $\sigma \in J_{\lambda}$ we have $|\operatorname{sn}(\sigma)|=\lambda^{-1 / 4}$. Hence

$$
\eta_{\sigma}:=\lambda^{1 / 4} \operatorname{sn} \sigma \quad \text { satisfies } \quad\left|\eta_{\sigma}\right|=1 .
$$


Proof. We write $\sigma=a+\frac{\mathrm{i}}{2} K^{\prime}(\lambda)$ for $a:=t K(\lambda)$ and all $t \in(-1,3)$. Hence,

$$
\operatorname{sn}(\sigma)=\operatorname{sn}\left(a+\frac{\mathrm{i}}{2} K^{\prime}(\lambda)\right) \stackrel{[1,16.5 .4,16.17 .1]}{=} \frac{\left(1+\lambda^{1 / 2}\right) \operatorname{sn} a+\mathrm{i} \operatorname{cn} a \operatorname{dn} a}{\lambda^{1 / 4}\left(1+\lambda^{1 / 2} \operatorname{sn}^{2} a\right)} .
$$

We have

$$
\begin{aligned}
& |\operatorname{sn}(\sigma)| \stackrel{[1,16.9 .1]}{=} \frac{\sqrt{\left(1+\lambda^{1 / 2}\right)^{2} \operatorname{sn}^{2} a+\left(1-\mathrm{sn}^{2} a\right)\left(1-\lambda \mathrm{sn}^{2} a\right)}}{\lambda^{1 / 4}\left(1+\lambda^{1 / 2} \mathrm{sn}^{2} a\right)}=\frac{\sqrt{2 \lambda^{1 / 2} \operatorname{sn}^{2} a+1+\lambda \mathrm{sn}^{4} a}}{\lambda^{1 / 4}\left(1+\lambda^{1 / 2} \operatorname{sn}^{2} a\right)} \\
& =\frac{1+\lambda^{1 / 2} \operatorname{sn}^{2} a}{\lambda^{1 / 4}\left(1+\lambda^{1 / 2} \operatorname{sn}^{2} a\right)}=\lambda^{-1 / 4} .
\end{aligned}
$$

Lemma 14 Let $\sigma \in J_{\lambda}$ and $\eta_{\rho}$ be as in (23). For any $0 \leq \rho \leq \pi / 6$ and $\zeta \in \mathbb{S}_{1}$ it holds

$$
\operatorname{sn}(\sigma+\rho \zeta)=\lambda^{-1 / 4} \eta_{\sigma}(1-\varepsilon),
$$

where $\varepsilon=\varepsilon_{\lambda}(\rho \zeta, \sigma)$ satisfies for all $0 \leq \rho \leq \pi / 6$ and $\zeta \in \mathbb{S}_{1}$

$$
\left|\varepsilon_{\lambda}(\rho \zeta, \sigma)\right| \leq 6\left(\rho+\left|\lambda^{1 / 2}-\eta_{\sigma}^{2}\right|\right) \rho .
$$

Proof. The addition formula $[1,16.17 .1]$ gives us

$$
\operatorname{sn}(\sigma+\rho \zeta)=\operatorname{sn}(\sigma)\left(1-\varepsilon_{\lambda}(\rho \zeta, \sigma)\right)
$$

where

$$
\varepsilon_{\lambda}(y, \sigma)=\frac{1-\operatorname{cn} y \operatorname{dn} y-\lambda^{1 / 2} \eta_{\sigma}^{2} \operatorname{sn}^{2} y-\eta_{\sigma}^{-1} \operatorname{sn} y \sqrt{\left(\lambda^{1 / 2}-\eta_{\sigma}^{2}\right)\left(1-\lambda^{1 / 2} \eta_{\sigma}^{2}\right)}}{1-\lambda^{1 / 2} \eta_{\sigma}^{2} \operatorname{sn}^{2} y} .
$$

The term $\varepsilon_{\lambda}(y, \sigma)$ can be estimated in the considered range of $\rho$ by using $[20$, Theorem 1]:

$$
|\operatorname{sn}(\rho \zeta)| \leq \tan \rho, \quad|\operatorname{cn}(\rho \zeta)| \leq \frac{1}{\cos \rho}, \quad|\operatorname{dn}(\rho \zeta)| \leq \frac{1}{\cos \rho} .
$$

Since

$$
1-\operatorname{cn}(\rho \zeta) \operatorname{dn}(\rho \zeta)=\operatorname{sn}^{2}(\rho \zeta) \frac{(1+\lambda)-\lambda \operatorname{sn}^{2}(\rho \zeta)}{1+\sqrt{\left(1-\operatorname{sn}^{2}(\rho \zeta)\right)\left(1-\lambda \mathrm{sn}^{2}(\rho \zeta)\right)}},
$$

we obtain for any $0 \leq \rho \leq \pi / 6$

$$
|1-\operatorname{cn}(\rho \zeta) \operatorname{dn}(\rho \zeta)| \leq \tan ^{2} \rho \frac{2}{1+\sqrt{\left(1-\tan ^{2} \rho\right)\left(1-\lambda \tan ^{2} \rho\right)}} \leq \frac{2 \tan ^{2} \rho}{2-\tan ^{2} \rho} .
$$


The other term in the numerator of (26) can be estimated by

$$
\left|\lambda^{1 / 2} \eta_{\sigma}^{2} \operatorname{sn}^{2} \rho \zeta\right|+\left|\eta_{\sigma}^{-1} \operatorname{sn} \rho \zeta \sqrt{\left(\lambda^{1 / 2}-\eta_{\sigma}^{2}\right)\left(1-\lambda^{1 / 2} \eta_{\sigma}^{2}\right)}\right| \leq \tan ^{2} \rho+\tan \rho \sqrt{\left|\lambda^{1 / 2}-\eta_{\sigma}^{2}\right|\left|1-\lambda^{1 / 2} \eta_{\sigma}^{2}\right|}
$$

Note that

$$
1-\lambda^{1 / 2} \eta_{\sigma}^{2}=1-\lambda+\lambda^{1 / 2}\left(\lambda^{1 / 2}-\eta_{\sigma}^{2}\right)
$$

and we use $\sqrt{a+b} \leq \sqrt{a}+\sqrt{b}$ for non-negative $a, b$ in order to estimate

$$
\left|\sqrt{\left(\lambda^{1 / 2}-\eta_{\sigma}^{2}\right)\left(1-\lambda^{1 / 2} \eta_{\sigma}^{2}\right)}\right| \leq 2 \sqrt{1-\lambda^{1 / 2}} \sqrt{\left|\lambda^{1 / 2}-\eta_{\sigma}^{2}\right|}+\left|\lambda^{1 / 2}-\eta_{\sigma}^{2}\right| \leq 3\left|\lambda^{1 / 2}-\eta_{\sigma}^{2}\right|,
$$

so that we obtain for $0 \leq \rho \leq \pi / 6$

$$
\left|\lambda^{1 / 2} \eta_{\sigma}^{2} \operatorname{sn}^{2} \rho \zeta\right|+\left|\eta_{\sigma}^{-1}(\operatorname{sn} \rho \zeta) \sqrt{\left(\lambda^{1 / 2}-\eta_{\sigma}^{2}\right)\left(1-\lambda^{1 / 2} \eta_{\sigma}^{2}\right)}\right| \leq \tan \rho\left(\tan \rho+3\left|\lambda^{1 / 2}-\eta_{\sigma}^{2}\right|\right)
$$

The denominator of (26) for $0 \leq \rho \leq \pi / 6$ can be estimated by

$$
\frac{1}{\left|1-\lambda^{1 / 2} \operatorname{sn}^{2} \rho \zeta\right|} \leq \frac{1}{1-\tan ^{2} \rho} \leq \frac{3}{2} .
$$

The combination of these estimates leads to

$$
\left|\varepsilon_{\lambda}(\rho \zeta, \sigma)\right| \leq \frac{3}{2}\left(\frac{11}{5} \tan ^{2} \rho+3\left|\lambda^{1 / 2}-\eta_{\sigma}^{2}\right| \tan \rho\right) .
$$

We employ $\tan \rho \leq 2 \frac{\sqrt{3}}{\pi} \rho$ for all $0 \leq \rho \leq \pi / 6$ (cf. (12)) to obtain

$$
\left|\varepsilon_{\lambda}(\rho \zeta, \sigma)\right| \leq 6\left(\rho+\left|\lambda^{1 / 2}-\eta_{\sigma}^{2}\right| \rho\right) \rho
$$

Lemma 15 The contour $\gamma_{M}(\sigma)$ (cf. Definition 5) is a parametrization of the circle $\mathcal{C}_{M}$ with radius $M$ about $M$ and can be written in the form

$$
\gamma_{M}(\sigma)=M\left(1+\frac{1-k^{1 / 2} \overline{\eta_{\sigma}}}{1-k^{1 / 2} \eta_{\sigma}} \eta_{\sigma}\right)
$$

with $\eta_{\sigma}$ as in (23) and $k$ as in (11). For the derivatives we get

$$
\left|\gamma_{M}^{\prime}(\sigma)\right| \leq 16 \lambda^{1 / 4} M \frac{\sqrt{2 q-1}}{q-1} \frac{\left|\lambda^{1 / 4}+\eta_{\sigma}\right|}{\left|\lambda^{1 / 4}-\eta_{\sigma}\right|} .
$$

Proof. We introduce the short hands

$$
u=\operatorname{sn} \sigma=\lambda^{-1 / 4} \eta_{\sigma}, \quad \lambda=k^{2}, \quad \nu=\sqrt{2 q-1}, \quad x=k^{1 / 2} \operatorname{Re} \eta_{\sigma}, \quad y=k^{1 / 2} \operatorname{Im} \eta_{\sigma} .
$$


Then, the definition of $z(u)$ (cf. (14)) leads to

$$
\begin{aligned}
z(u)-M & =\frac{M}{(q-1)} \frac{\nu-q+(\nu+q) k^{1 / 2} \eta_{\sigma}}{1-k^{1 / 2} \eta_{\sigma}} \\
& =\frac{M(q+\nu)}{q-1} \frac{x-k+\mathrm{i} y}{1-x-\mathrm{i} y}=\frac{M}{\sqrt{k}} \frac{x-k+\mathrm{i} y}{1-x-\mathrm{i} y}=M \eta_{\sigma} \frac{1-k^{1 / 2} \overline{\eta_{\sigma}}}{1-k^{1 / 2} \eta_{\sigma}} .
\end{aligned}
$$

From

$$
\left|\frac{x-k+\mathrm{i} y}{1-x-\mathrm{i} y}\right|=\sqrt{\frac{(x-k)^{2}+y^{2}}{(1-x)^{2}+y^{2}}}=\sqrt{k}
$$

we conclude that $|z(u)-M|=M$, i.e., $\gamma_{M}(\sigma)$ is a parametrization of $\mathcal{C}_{M}$.

For the derivative $\gamma_{M}^{\prime}(\sigma)$ we obtain

$\gamma_{M}^{\prime}(\sigma) \stackrel{[1,16.16 .1]}{=} M \frac{\sqrt{2 q-1}}{q-1} \frac{2 \lambda^{1 / 2} \operatorname{cn}(\sigma) \operatorname{dn}(\sigma)}{\left(\lambda^{1 / 2} \operatorname{sn}(\sigma)-1\right)^{2}}=M \frac{\sqrt{2 q-1}}{q-1} \frac{2 \lambda^{1 / 2} \sqrt{\left(1-\lambda^{-1 / 2} \eta_{\sigma}^{2}\right)\left(1-\lambda^{1 / 2} \eta_{\sigma}^{2}\right)}}{\left(\lambda^{1 / 4} \eta_{\sigma}-1\right)^{2}}$.

In order to prove estimate (28) we need Lemma 16 below.

Next, we will estimate the derivative $\gamma_{M}^{\prime}$ in a neighborhood of $J_{\lambda}$.

Lemma 16 For $\sigma \in J_{\lambda}$ it holds

$$
\left|\gamma_{M}^{\prime}(\sigma+\rho \zeta)\right|=\gamma_{M}^{\prime}(\sigma)+\rho \text { Rest },
$$

for all $\rho \leq \frac{\left|\lambda^{1 / 2}-\eta_{\sigma}^{2}\right|^{1 / 2}}{40}$ and all $\zeta \in \mathbb{S}_{1}$, where

$$
\mid \text { Rest } \mid \leq 184 \frac{\sqrt{2 q-1}}{q-1} \frac{M}{\left|\lambda^{1 / 4}-\eta_{\sigma}\right|^{2}} .
$$

Proof. Let $\sigma \in J_{\lambda}$. The first derivative of $\gamma_{M}$ can be written in the form

$$
\gamma_{M}^{\prime}(\sigma+\rho \zeta) \stackrel{[1,16.16 .1]}{=} M \frac{\sqrt{2 q-1}}{q-1} \frac{2 \lambda^{1 / 2} \operatorname{cn}(\sigma+\rho \zeta) \operatorname{dn}(\sigma+\rho \zeta)}{\left(\lambda^{1 / 2} \operatorname{sn}(\sigma+\rho \zeta)-1\right)^{2}} .
$$

We use (24) and write

$$
x:=x(\varepsilon):=\operatorname{sn}(\sigma+\rho \zeta)=\eta_{\sigma} \frac{1-\varepsilon}{\lambda^{1 / 4}},
$$

so that

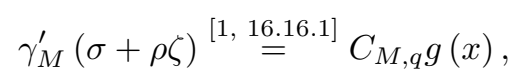

where $C_{M, q}:=2 M \lambda^{1 / 2} \frac{\sqrt{2 q-1}}{q-1}$ and

$g(x):=g_{1}(x) g_{2}(x) \quad$ and $\quad g_{1}(x):=\sqrt{\left(1-x^{2}\right)\left(1-\lambda x^{2}\right)} \quad$ and $\quad g_{2}(x):=\left(1-\lambda^{1 / 2} x\right)^{-2}$. 
We obtain for $0 \leq \rho \leq \frac{\left|\lambda^{1 / 2}-\eta_{\sigma}^{2}\right|^{1 / 2}}{40}$ the estimate

$$
|\varepsilon| \leq 6\left(\rho+\left|\lambda^{1 / 2}-\eta_{\sigma}^{2}\right|\right) \rho \leq \frac{1}{4}\left|\lambda^{1 / 2}-\eta_{\sigma}^{2}\right| \quad \text { and } \quad|\varepsilon| \leq 1 / 2 .
$$

For the functions $g_{2}, g_{2}^{\prime}$ we need some auxiliary estimates. The modulus of $x$ can be estimated by

$$
\frac{1}{2 \lambda^{1 / 4}} \leq|x| \leq \frac{3}{2 \lambda^{1 / 4}}
$$

Then,

$$
\begin{aligned}
\left|1-\lambda^{1 / 2} x\right| & =\left|1-\lambda^{1 / 4} \eta_{\sigma}(1-\varepsilon)\right|=\left|1-\lambda^{1 / 4} \eta_{\sigma}+\lambda^{1 / 4} \eta_{\sigma} \varepsilon\right| \\
& \geq\left|1-\lambda^{1 / 4} \eta_{\sigma}\right|-\lambda^{1 / 4}|\varepsilon| \geq\left|1-\lambda^{1 / 4} \eta_{\sigma}\right|-\lambda^{1 / 4} \frac{1}{4}\left|\lambda^{1 / 2}-\eta_{\sigma}^{2}\right| .
\end{aligned}
$$

Note that $\left|1-\lambda^{1 / 4} \eta_{\sigma}\right|=\left|\lambda^{1 / 4}-\eta_{\sigma}\right|$ so that

$$
\left|1-\lambda^{1 / 2} x\right| \geq\left|\lambda^{1 / 4}-\eta_{\sigma}\right|\left(1-\frac{1}{4}\left|\lambda^{1 / 4}+\eta_{\sigma}\right|\right) \geq \frac{1}{2}\left|\lambda^{1 / 4}-\eta_{\sigma}\right| .
$$

Thus,

$$
\left|g_{2}(x)\right| \leq \frac{4}{\left|\lambda^{1 / 4}-\eta_{\sigma}\right|^{2}} \quad \text { and } \quad\left|g_{2}^{\prime}(x)\right| \leq \frac{2}{\left|1-\lambda^{1 / 2} x\right|^{3}} \leq \frac{16}{\left|\lambda^{1 / 4}-\eta_{\sigma}\right|^{3}} .
$$

The estimate of the first derivative of $g_{1}$ is more involved. Explicit calculation leads to

$$
g_{1}^{\prime}(x)=-x\left(\sqrt{\frac{1-\lambda x^{2}}{1-x^{2}}}+\lambda \sqrt{\frac{1-x^{2}}{1-\lambda x^{2}}}\right) .
$$

Similarly as for (32) we get

$$
\left|1+\lambda^{1 / 2} x\right| \geq \frac{1}{2}\left|\lambda^{1 / 4}+\eta_{\sigma}\right|
$$

so that the combination of (32) and (35) yields

$$
\left|1-\lambda x^{2}\right| \geq \frac{1}{4}\left|\lambda^{1 / 2}-\eta_{\sigma}^{2}\right| .
$$

For the lower estimate of $\left|1-x^{2}\right|$ we argue as follows. It holds

$$
\begin{aligned}
\left|1-x^{2}\right| & =\left|1-\eta_{\sigma}^{2} \frac{(1-\varepsilon)^{2}}{\lambda^{1 / 2}}\right|=\lambda^{-1 / 2}\left|\lambda^{1 / 2}-\eta_{\sigma}^{2}(1-\varepsilon)^{2}\right| \\
& \geq \lambda^{-1 / 2}\left(\left|\lambda^{1 / 2}-\eta_{\sigma}^{2}\right|-|\varepsilon(2-\varepsilon)|\right) .
\end{aligned}
$$


Since $0 \leq \rho \leq \frac{\left|\lambda^{1 / 2}-\eta_{\sigma}^{2}\right|^{1 / 2}}{40}$ and $\left|\lambda^{1 / 2}-\eta_{\sigma}^{2}\right| \leq \sqrt{2}\left|\lambda^{1 / 2}-\eta_{\sigma}^{2}\right|^{1 / 2}$, we get from (30) the estimates

$$
|\varepsilon| \leq \frac{1}{4}\left|\lambda^{1 / 2}-\eta_{\sigma}^{2}\right| \quad \text { and } \quad|\varepsilon(2-\varepsilon)| \leq \frac{1}{2}\left|\lambda^{1 / 2}-\eta_{\sigma}^{2}\right|
$$

so that

$$
\left|1-x^{2}\right| \geq \frac{\left|\lambda^{1 / 2}-\eta_{\sigma}^{2}\right|}{2 \lambda^{1 / 2}} .
$$

The estimates of $\left|1-\lambda x^{2}\right|$ and $\left|1-x^{2}\right|$ from above follow from

$$
\begin{aligned}
\left|1-\lambda^{1 / 2} x\right| & \leq\left|1-\lambda^{1 / 4} \eta_{\sigma}\right|+\lambda^{1 / 4}|\varepsilon| \leq\left|1-\lambda^{1 / 4} \eta_{\sigma}\right|+\lambda^{1 / 4} \frac{1}{4}\left|\lambda^{1 / 2}-\eta_{\sigma}^{2}\right| \\
& \leq\left|\lambda^{1 / 4}-\eta_{\sigma}\right|\left(1+\lambda^{1 / 4} \frac{1}{4}\left|\lambda^{1 / 4}+\eta_{\sigma}\right|\right) \leq \frac{3}{2}\left|\lambda^{1 / 4}-\eta_{\sigma}\right| .
\end{aligned}
$$

Replacing $\eta_{\sigma}$ by $-\eta_{\sigma}$ leads to

$$
\left|1+\lambda^{1 / 2} x\right| \leq \frac{3}{2}\left|\lambda^{1 / 4}+\eta_{\sigma}\right|
$$

and, in turn,

$$
\left|1-\lambda x^{2}\right| \leq \frac{9}{4}\left|\lambda^{1 / 2}-\eta_{\sigma}^{2}\right|
$$

To estimate $\left|1-x^{2}\right|$ from above we argue as in (37), (38) to obtain

$$
\left|1-x^{2}\right| \leq \frac{3}{2 \lambda^{1 / 2}}\left|\lambda^{1 / 2}-\eta_{\sigma}^{2}\right| .
$$

The combination of (31), (34), (36), (39), (40), and (41) results in

$$
\left|g_{1}^{\prime}(x)\right| \leq 7 .
$$

It remains to estimate $g_{1}(x)$. We use (40) and (41) to obtain

$$
\left|g_{1}(x)\right| \leq \sqrt{\left|1-x^{2}\right|\left|1-\lambda x^{2}\right|} \leq 2 \frac{\left|\lambda^{1 / 2}-\eta_{\sigma}^{2}\right|}{\lambda^{1 / 4}} .
$$

In total, we have proved

$$
\gamma_{M}^{\prime}(\sigma+\rho \zeta)=\gamma_{M}^{\prime}(\sigma)+\rho \text { Rest },
$$

where

$$
\begin{aligned}
\mid \text { Rest } \mid & \leq \max _{\substack{0 \leq \mu \leq \rho \\
|\zeta|=1}}\left|\gamma_{M}^{\prime \prime}(\sigma+\mu \zeta)\right| \leq C_{M, q} \max _{\substack{|\varepsilon| \leq \frac{1}{4}\left|\lambda^{1 / 2}-\eta_{\sigma}^{2}\right| \\
x=\eta_{\sigma} \frac{1-\varepsilon}{\lambda^{1 / 4}}}}\left|g_{1}^{\prime}(x) g_{2}(x)+g_{1}(x) g_{2}^{\prime}(x)\right| \\
& \leq 184 \lambda^{1 / 4} M \frac{\sqrt{2 q-1}}{q-1} \frac{1}{\left|\lambda^{1 / 4}-\eta_{\sigma}\right|^{2}} .
\end{aligned}
$$


Proof of estimate (28).

Note that $\rho=0$ implies $\varepsilon=0$ (in (24)) and, in turn $x=\lambda^{-1 / 4} \eta_{\sigma}$. We have

$$
\gamma_{M}^{\prime}(\sigma)=C_{M, q} g\left(\lambda^{-1 / 4} \eta_{\sigma}\right) .
$$

From (42) and (33) we get

$$
\left|\gamma_{M}^{\prime}(\sigma)\right| \leq 16 \lambda^{1 / 4} M \frac{\sqrt{2 q-1}}{q-1} \frac{\left|\lambda^{1 / 4}+\eta_{\sigma}\right|}{\left|\lambda^{1 / 4}-\eta_{\sigma}\right|} .
$$

Corollary 17 For all $0 \leq \rho \leq \frac{q^{-1 / 4}}{40}$, it holds

$$
\left|\gamma_{M}^{\prime}(\sigma)\right| \stackrel{(12)}{\leq} M \frac{\sqrt{2 q-1}}{q-1}(64 \sqrt{q}+736 q \rho) \quad \forall \sigma \in \mathfrak{s}_{\rho}\left(J_{\lambda}\right) .
$$

Proof. It is easy to verify (cf. (12)) that the condition on $\rho$ implies

$$
\rho \leq \frac{\sqrt{\left|\lambda^{1 / 2}-\eta_{\sigma}^{2}\right|}}{40} \quad \forall \sigma \in J_{\lambda}
$$

and, hence, Lemma 16 is applicable. We employ estimate (28) and Lemma 16 to obtain

$$
\begin{aligned}
\left|\gamma_{M}^{\prime}(\sigma+\rho \zeta)\right| & \leq M \frac{\sqrt{2 q-1}}{q-1}\left(16 \lambda^{1 / 4} \frac{\left|\lambda^{1 / 4}+1\right|}{\left|\lambda^{1 / 4}-1\right|}+184 \frac{\rho}{\left|\lambda^{1 / 4}-1\right|^{2}}\right) \\
& \stackrel{(12)}{\leq} M \frac{\sqrt{2 q-1}}{q-1}(64 \sqrt{q}+736 q \rho) .
\end{aligned}
$$

Corollary 18 Let $0 \leq \rho \leq \frac{q^{-1 / 4}}{40}$. Then, the distance of the boundary of the region $\mathcal{C}_{M, \rho}$ to the contour $\mathcal{C}_{M}$ can be estimated by

$\left|\gamma_{M}(\sigma+\rho \zeta)-\gamma_{M}(\sigma)\right| \leq 92 M \frac{\sqrt{2 q-1}}{q-1} \rho\left(\frac{\left|\lambda^{1 / 2}-\eta_{\sigma}^{2}\right|+\rho}{\left|\lambda^{1 / 4}-\eta_{\sigma}\right|^{2}}\right) \quad \forall \sigma \in J_{\lambda} \quad$ and $\quad \zeta \in \mathbb{S}_{1}$.

Proof. Again, the condition on $\rho$ implies (45) and Lemma 16 is applicable. We have

$$
\begin{aligned}
\left|\gamma_{M}(\sigma+\rho \zeta)-\gamma_{M}(\sigma)\right| & \stackrel{(43)}{\leq} \rho\left|\gamma_{M}^{\prime}(\sigma)\right|+\frac{\rho^{2}}{2} \mid \text { Rest } \mid \\
& \stackrel{(28),(44)}{\leq} 92 M \frac{\sqrt{2 q-1}}{q-1} \rho\left(\frac{\left|\lambda^{1 / 2}-\eta_{\sigma}^{2}\right|+\rho}{\left|\lambda^{1 / 4}-\eta_{\sigma}\right|^{2}}\right) .
\end{aligned}
$$

In the following the width of $\mathcal{C}_{M, \rho}$ will be estimated in terms of $z \in \mathcal{C}_{M}$. 
Corollary 19 Let $0 \leq \rho \leq \frac{q^{-1 / 4}}{40}$. For any $z=M(1+\theta), \theta \in \mathbb{S}_{1}$, the width of $\mathcal{C}_{M, \rho}$ is bounded by

$$
\operatorname{dist}\left(z, \partial C_{M, \rho}\right) \leq 261 \frac{M \rho}{q-1}\left(q|1+\theta|+1+\left(q^{3 / 2}|1+\theta|^{2}+q^{1 / 2}\right) \rho\right) .
$$

Proof. From (27) we conclude that any point on $\mathcal{C}_{M}$ can be written as $z=$ $M(1+\theta)$ for $\theta \in \mathbb{S}_{1}$ and corresponds to

$$
\eta_{\sigma}=\frac{\theta+k^{1 / 2}}{1+k^{1 / 2} \theta}
$$

We combine (46) and (48) to bound the width of $\mathcal{C}_{M, \rho}$ for any $z=M(1+\theta)$ by

$$
\operatorname{dist}\left(z, \partial C_{M, \rho}\right) \leq 92 M \frac{\sqrt{2 q-1}}{q-1} \rho\left(\frac{\left|2 k^{1 / 2}+(k+1) \theta\right|}{1-k}+\frac{\left|1+k^{1 / 2} \theta\right|^{2} \rho}{(1-k)^{2}}\right)
$$

For the first term in the bracket, we get with $\nu$ as in (29)

$$
\frac{\left|2 k^{1 / 2}+k \theta+\theta\right|}{1-k}=\frac{|q(1+\theta)-1|}{\nu} \leq \frac{q|1+\theta|+1}{\nu}
$$

and for the second one

$$
\frac{\left|1+k^{1 / 2} \theta\right|^{2} \rho}{(1-k)^{2}}=\frac{|q(1+\theta)+\nu-\theta|^{2} \rho}{4 \nu^{2}} \leq\left(q^{2}|1+\theta|^{2}+(\nu+1)^{2}\right) \frac{\rho}{2 \nu^{2}} .
$$

This leads to

$$
\operatorname{dist}\left(z, \partial C_{M, \rho}\right) \leq 92 M \frac{1}{q-1} \rho\left(q|1+\theta|+1+\left(q^{3 / 2}|1+\theta|^{2}+\sqrt{2} q^{1 / 2}\right) 2 \rho\right) .
$$

The following theorem estimates how far the contour $\gamma_{M}(\sigma+\mathrm{i} \rho)$ enters the left half plane. The title of the Theorem is motivated by the shape of the outer contour, as depicted in Figure 1.

Theorem 20 (Kidney Distance) Let $c_{0} \in(0,1), m, M$, and $q$ be as in (2). For any $\rho \leq\left(3 C_{0}\right)^{-1} \min \left\{M^{-1 / 2}, m^{-1}\right\}$ with $C_{0}=\frac{370}{c_{0}}$, it holds for $\mu$ as in (9)

$$
\mu(M, \rho) \geq-1
$$

Proof. For $\theta \in \mathbb{S}_{1}$, we define $x_{\theta}:=1+\operatorname{Re} \theta$ and $y_{\theta}:=\operatorname{Im} \theta$ and note that $M\left(x_{\theta}+\mathrm{i} y_{\theta}\right) \in \mathcal{C}_{M}, x_{\theta} \in[0,2]$ and $y_{\theta} \in[-1,1]$. The right-hand side in (47) defines a function $d_{\lambda, M, q, \rho}(\theta)$ and we write short $d(\theta)$ if there is no confusion. It holds

$$
\mu(M, \rho) \geq \min _{\theta \in \mathbb{S}_{1}}\left\{M x_{\theta}-d(\theta)\right\}
$$


and we will estimate the right-hand side in (49) from below. The relation $\left(x_{\theta}-1\right)^{2}+y_{\theta}^{2}=1$ implies $|1+\theta|^{2}=x_{\theta}^{2}+y_{\theta}^{2}=2 x_{\theta}$ and we obtain the estimate

$$
d(\theta) \leq C_{0} M \rho\left(\sqrt{x_{\theta}}+q^{-1}+\left(q^{1 / 2} x_{\theta}+q^{-1 / 2}\right) \rho\right) \quad \text { with } \quad C_{0}:=\frac{370}{c_{0}} .
$$

For $0 \leq x_{\theta} \leq 1 / M$ and $0 \leq \rho \leq \frac{M^{-1 / 2}}{3 C_{0}}$ we get

$$
3 \rho \sqrt{x_{\theta}} \leq \frac{1}{C_{0} M} .
$$

For $1 / M \leq x_{\theta} \leq 2$ and, again, $0 \leq \rho \leq \frac{M^{-1 / 2}}{3 C_{0}}$ it holds

$$
3 \rho \sqrt{x_{\theta}} \leq 3 \rho \frac{x_{\theta}}{\sqrt{x_{\theta}}} \leq 3 \rho \sqrt{M} x_{\theta} \leq \frac{x_{\theta}}{C_{0}}
$$

so that for all $x_{\theta}$ and $0 \leq \rho \leq \frac{M^{-1 / 2}}{3 C_{0}}$ we have shown

$$
\rho \sqrt{x_{\theta}} \leq \frac{1}{3 C_{0}}\left(x_{\theta}+\frac{1}{M}\right) .
$$

For the second term in the right-hand side in (50) we get for $0 \leq \rho \leq \frac{1}{3 m C_{0}}$ the estimate

$$
\frac{\rho}{q} \leq \frac{1}{3 C_{0} M} \leq \frac{1}{3 C_{0}}\left(x_{\theta}+\frac{1}{M}\right) .
$$

The last bracket of the right-hand side in (50) can be estimated for $0 \leq \rho \leq$ $\frac{M^{-1 / 2}}{3 C_{0}}$ by

$$
\begin{aligned}
\left(q^{1 / 2} x_{\theta}+q^{-1 / 2}\right) \rho^{2} & \leq \frac{1}{\left(3 C_{0}\right)^{2}}\left(\frac{1}{\sqrt{m M}} x_{\theta}+\sqrt{\frac{m}{M}} \frac{1}{M}\right) \\
& \stackrel{(2 a))}{\leq} \frac{1}{\left(3 C_{0}\right)^{2}}\left(x_{\theta}+\frac{1}{M}\right) \leq \frac{1}{3 C_{0}}\left(x_{\theta}+\frac{1}{M}\right) .
\end{aligned}
$$

In total we have proved that the function $d(\theta)$ can be estimated by

$$
d(\theta) \leq M x_{\theta}+1 .
$$

Thus,

$$
\mu_{M, \rho} \geq \min _{\theta \in \mathbb{S}_{1}}\left\{M x_{\theta}-d(\theta)\right\} \geq-1 .
$$

The following Theorem estimates how far the contour $\gamma_{M}(\sigma-i \rho)$ moves towards the interval $\mathcal{I}=[m, M]$ containing the poles of our integrand in (3). The title of the theorem is again motivated by the shape of this contour, see Figure 1. 
Theorem 21 (Egg Distance) Let $A \in[m, M]$. For $\rho \leq\left(3 C_{0}\right)^{-1} \min \left\{M^{-1 / 2}, m^{-1}\right\}$ we have

$$
\operatorname{dist}\left(A, \mathcal{C}_{M, \rho}\right) \geq\left(1-2 M^{-1 / 2}-\frac{2}{3} m^{-1}\right) A .
$$

Proof. We write $A=M \xi$ for $\xi \in\left[q^{-1}, 1\right]$. The distance of $A$ to the contour can be bounded from below by

$$
\operatorname{dist}\left(M \xi, \mathcal{C}_{M, \rho}\right) \geq \min _{\theta \in \mathbb{S}_{1}}|M(1+\theta)-d(\theta) \theta-M \xi| .
$$

With the choice $0 \leq \rho \leq\left(3 C_{0}\right)^{-1} \min \left\{M^{-1 / 2}, m^{-1}\right\}$ we obtain for the distance function and $x_{\theta}$ as in the proof of Theorem 20

$$
d(\theta) \leq \frac{2}{3}\left(\sqrt{M x_{\theta}}+1\right) .
$$

Note that

$|M(1+\theta)-d(\theta) \theta-M \xi| \geq M|1+\theta-\xi|-|d(\theta)| \geq M \sqrt{\xi^{2}+2 x_{\theta}(1-\xi)}-\frac{2}{3}\left(\sqrt{M x_{\theta}}+1\right)$.

With the aid of the symbolic algebra program MATHEMATICA, we find that the right-hand side takes a minimum as a function of $x_{\theta} \in[0,2]$ at

$$
x_{\theta}=\left\{\begin{array}{cl}
2 & \text { if } 1-\frac{1}{\sqrt{18 M}-1} \leq \xi \leq 1 \\
\frac{\xi^{2}}{(9 M(1-\xi)-2)(1-\xi)} & \text { if } 0 \leq \xi \leq 1-\frac{1}{\sqrt{18 M}-1} .
\end{array}\right.
$$

Hence, after some manipulations we get

$$
|M(1+\theta)-d(\theta) \theta-M \xi| \geq \begin{cases}M(2-\xi)-2 \sqrt{M} & \text { if } 1-\frac{1}{\sqrt{18 M}-1} \leq \xi \leq 1 \\ (M-2 \sqrt{M}) \xi-\frac{2}{3} & \text { if } 0 \leq \xi \leq 1-\frac{1}{\sqrt{18 M}-1}\end{cases}
$$

Since $\xi \geq q^{-1}$ we have $2 / 3 \leq(2 / 3) \xi q$ and the assertion follows.

Corollary 22 For $\rho \leq\left(3 C_{0}\right)^{-1} \min \left\{M^{-1 / 2}, m^{-1}\right\}$ we have

$$
\max \left\{|z|: z \in \mathcal{C}_{M, \rho}\right\} \leq \frac{10}{3} M .
$$

Proof. The assertion follows from (51) and $\max \left\{|z|: z \in \mathcal{C}_{M}\right\}=2 M$.

In order to estimate the length of the interval $J_{\lambda}$ we need to estimate the complete elliptic integral.

Lemma 23 The complete elliptic integral $K$ is strictly monotonously increasing in $[0,1[$ and satisfies the estimate

$$
\frac{\pi}{2} \leq K(\lambda) \leq \frac{1}{2} \log \frac{16}{1-\lambda}\left(1+\sqrt{\frac{2}{7}}(1-\lambda)\right) \quad \forall \lambda \in[0,1[.
$$


Proof. The strict monotonicity and the endpoint value at $\lambda=0$ follow directly from (10a).

From [11, 8.113 (3) (with the substitution $k^{2} \leftarrow \lambda$ therein)] we obtain

$$
\begin{aligned}
K(\lambda) & =\sum_{\ell=0}^{\infty}\left(\frac{(2 \ell) !}{(\ell !)^{2}}\right)^{2}\left(\frac{1-\lambda}{16}\right)^{\ell}\left(\log \frac{4}{\sqrt{1-\lambda}}-2 \sum_{m=1}^{\ell} \frac{1}{(2 m-1) 2 m}\right) \\
& =\frac{1}{2} \log \frac{16}{1-\lambda}+\sum_{\ell=1}^{\infty}\left(\frac{(2 \ell) !}{\ell !^{2}}\right)^{2}\left(\frac{1-\lambda}{16}\right)^{\ell}\left(\frac{1}{2} \log \frac{16}{1-\lambda}-2 \sum_{m=1}^{\ell} \frac{1}{(2 m-1) 2 m}\right) .
\end{aligned}
$$

By using

$$
\sum_{m=1}^{\infty} \frac{1}{(2 m-1) 2 m}=\log 2
$$

we get

$$
|K(\lambda)| \leq \frac{1}{2} \log \frac{16}{1-\lambda}\left(1+\frac{1-\lambda}{8} \sum_{\ell=1}^{\infty}\left(\frac{(2 \ell) !}{\ell !^{2}}\right)^{2}\left(\frac{1-\lambda}{16}\right)^{\ell-1}\right) .
$$

The infinite sum on the right-hand side is monotonously decreasing for $\lambda \in] 0,1]$. Hence, for $\lambda \in\left[\frac{1}{2}, 1\right]$ we have

$$
|K(\lambda)| \leq \frac{1}{2} \log \frac{16}{1-\lambda}\left(1+\frac{1-\lambda}{8} \sum_{\ell=1}^{\infty} \frac{(2 \ell) !}{(\ell !)^{2}}\left(\frac{1}{32}\right)^{\ell-1}\right) \leq \frac{1}{2} \log \frac{16}{1-\lambda}\left(1+\sqrt{\frac{2}{7}}(1-\lambda)\right) .
$$

The definition (10a) shows that $K(\lambda)$ is strictly monotonously increasing in $\lambda \in[0,1]$ so that estimate (52) holds for all $\lambda \in[0,1[$.

\section{$5 \quad$ Numerical Experiments}

As it is common for developments in the field of numerical quadrature, new challenges arise from new types of applications. Besides the classical Newton expansion of an interpolating polynomial, high order divided differences arise when using variable time stepping in the generalized convolution quadrature method (GCQ) for approximating convolution operators. The quadrature problem is particularly challenging if the application is related to the retarded potential boundary integral equations (RPBIE) for solving the three-dimensional wave and Maxwell equation in exterior domains (see [15]).

The application of the GCQ to the wave equation leads to an integral operator valued integrand $\mathcal{K}$ in (4), i.e., $f \leftarrow \mathcal{K}$, which is a function of the frequency variable $z \in \mathbb{C}$. From, e.g., [15, (3), (9), Proposition 8], it follows that the function

$$
f(z)=(1+z)^{4} \mathrm{e}^{-z}
$$

reflects the characteristic (spectral) properties of the operator valued function $\mathcal{K}$ : a) exponential growth to the left half plane, b) polynomial growth to the 
right, and c) an oscillatory behavior for increasing imaginary part. We will use this function $f$ for our numerical experiments and refer to [16] for the application to the wave equation. Our choice of nodes $x_{\ell}$ is also related to the variable time stepping in the GCQ method. If the solution of the retarded potential integral equation (or its derivative) has a singularity, say, at time $t=0$, then, from the approximation point of view, an algebraic grading of the time steps towards the origin can properly resolve the singularity. This consideration is reflected by our choice

$$
t_{\ell}=\left(\frac{\ell}{N}\right)^{\alpha}, \quad \Delta_{\ell}=t_{\ell+1}-t_{\ell}, \quad \text { for } \ell=0,1, \ldots
$$

with the grading exponent $\alpha ; \alpha=1$ corresponds to uniform time steps while $\alpha=$ 2 is a typical choice for resolving a qualitative behavior $O\left(t^{1 / 2}\right)$ of the solution at the origin. The arising divided differences are related to the reciprocal mesh sizes (cf. [15]), more precisely, are given by

$$
x_{\ell}=\frac{1}{\Delta_{\ell}}, \quad \text { for } \quad \forall \ell=1, \ldots, N
$$

and our goal is to approximate the scaled divided differences

$$
\begin{aligned}
\omega_{1, N}(0) \frac{1}{2 \pi \mathrm{i}}\left[x_{1}, \ldots, x_{N}\right] f & =\prod_{\ell=1}^{N}\left(-x_{\ell}\right) \frac{1}{2 \pi \mathrm{i}} \int_{\mathcal{C}_{M}} \frac{f(z)}{\prod_{\ell=1}^{N}\left(z-x_{\ell}\right)} d z \\
& =\frac{1}{2 \pi \mathrm{i}} \int_{\mathcal{C}_{M}} \frac{f(z)}{\prod_{\ell=1}^{N}\left(1-\frac{z}{x_{\ell}}\right)} d z
\end{aligned}
$$

by our new contour quadrature. With this notation (55) becomes

$$
\frac{1}{2 \pi \mathrm{i}} \int_{\mathcal{C}_{M}} \frac{f(z)}{\prod_{\ell=1}^{N}\left(1-\Delta_{\ell} z\right)} d z
$$

The computation of "exact" solutions for this experiments is not a trivial task and we employed the software MATHEMATICA, which allows to work with arbitrary high precision. Even though, we were not able to compute reliable reference solutions with MATHEMATICA for very high order divided differences for all values of $\alpha$, in particular for $\alpha$ in between 0.5 and 1.8. In these cases, we have computed a reference solution with our method by using $\max \left\{3 \cdot 10^{5}, 2 N^{2}\right\}$ quadrature points.

Experiment 1: Performance for Quadratic Mesh Grading Relative errors for different values of $N_{Q}$ and $N$ are provided in Table 1.

The upper half of the table, i.e., $N_{Q} \in[20,320]$, shows the fast convergence with respect to $N_{Q}$ and, in addition, that the convergence starts later for higher order divided differences. We also see that for $N_{Q}<N$ the accuracy becomes unreliable and the asymptotic convergence is not yet reached. 


\begin{tabular}{|c|c|c|c|c|c|}
\hline \multicolumn{7}{|c|}{$\alpha=2$} \\
\hline \hline$N_{Q}$ & $N=8$ & $N=32$ & $N=128$ & $N=512$ & $N=2048$ \\
\hline 20 & $2.1502 \mathrm{e}-1$ & 3.5308 & 1.4086 & 1.0001 & 1.0000 \\
40 & $3.7623 \mathrm{e}-3$ & $\frac{5.9599 \mathrm{e}-2}{2.0090 \mathrm{e}-1}$ & $4.4724 \mathrm{e}-1$ & 1.0000 \\
80 & $7.3213 \mathrm{e}-9$ & $4.2988 \mathrm{e}-5$ & $2.2862 \mathrm{e}-3$ & $3.0797 \mathrm{e}-1$ & $2.8917 \mathrm{e}-1$ \\
160 & $2.5628 \mathrm{e}-15$ & $4.2252 \mathrm{e}-10$ & $\underline{9.9688 \mathrm{e}-12}$ & $1.5785 \mathrm{e}-2$ & $7.9961 \mathrm{e}-1$ \\
320 & $2.2203 \mathrm{e}-16$ & $3.2376 \mathrm{e}-15$ & $4.8974 \mathrm{e}-15$ & $5.8516 \mathrm{e}-11$ & $7.7515 \mathrm{e}-2$ \\
\hline \hline$N$ & 3.4100 & $3.8665 \mathrm{e}-1$ & $1.8754 \mathrm{e}-8$ & $3.6240 \mathrm{e}-15$ & $1.0184 \mathrm{e}-14$ \\
$N \log (N)$ & $3.3270 \mathrm{e}-1$ & $3.5767 \mathrm{e}-8$ & $5.0626 \mathrm{e}-16$ & $7.2342 \mathrm{e}-15$ & $5.5631 \mathrm{e}-15$ \\
$N(\log (N))^{2}$ & $3.7623 \mathrm{e}-3$ & $1.7210 \mathrm{e}-15$ & $1.7291 \mathrm{e}-15$ & $5.2064 \mathrm{e}-16$ & $3.7840 \mathrm{e}-15$ \\
$N^{2}$ & $3.5023 \mathrm{e}-6$ & $1.9752 \mathrm{e}-15$ & $8.7585 \mathrm{e}-16$ & $4.1947 \mathrm{e}-15$ & $5.5929 \mathrm{e}-14$ \\
\hline
\end{tabular}

Table 1: Computation of the integral in (56) for $x_{\ell}$ in (54) with grading factor $\alpha=2$. We have underlined in each column the first number, where $N_{Q} \geq N$ is satisfied (cf. Corollary 12; note that $m=O(N)$ holds in the considered case). Note that the working precision in our MATLAB implementation is about $10^{-16}$.

\begin{tabular}{|c|c|c|c|c|c|}
\hline \multicolumn{7}{|c|}{$\alpha=1.1$} \\
\hline \hline$N_{Q}$ & $N=8$ & $N=32$ & $N=128$ & $N=512$ & $N=2048$ \\
\hline 20 & $\underline{4.2110 \mathrm{e}-7}$ & $1.5008 \mathrm{e}-1$ & $8.8498 \mathrm{e}-1$ & $4.1609 \mathrm{e}-1$ & 1.0000 \\
40 & $2.2196 \mathrm{e}-14$ & $\underline{1.2963 \mathrm{e}-9}$ & $9.5680 \mathrm{e}-2$ & $9.0889 \mathrm{e}-1$ & $4.9303 \mathrm{e}-1$ \\
80 & $2.2196 \mathrm{e}-14$ & $1.2434 \mathrm{e}-14$ & $1.7946 \mathrm{e}-9$ & $1.0042 \mathrm{e}-1$ & $9.7628 \mathrm{e}-1$ \\
160 & $2.2201 \mathrm{e}-14$ & $1.4694 \mathrm{e}-14$ & $\underline{4.2353 \mathrm{e}-14}$ & $8.7141 \mathrm{e}-9$ & $1.1488 \mathrm{e}-1$ \\
320 & $2.2196 \mathrm{e}-14$ & $1.3668 \mathrm{e}-14$ & $4.2508 \mathrm{e}-14$ & $2.1433 \mathrm{e}-14$ & $2.8493 \mathrm{e}-8$ \\
\hline \hline$N$ & $1.7713 \mathrm{e}-1$ & $3.7742 \mathrm{e}-5$ & $4.0800 \mathrm{e}-14$ & $2.5120 \mathrm{e}-14$ & $1.5834 \mathrm{e}-13$ \\
$N \log (N)$ & $2.6919 \mathrm{e}-11$ & $1.3043 \mathrm{e}-14$ & $4.2679 \mathrm{e}-14$ & $2.6638 \mathrm{e}-14$ & $1.6050 \mathrm{e}-13$ \\
$N(\log (N))^{2}$ & $2.2196 \mathrm{e}-14$ & $1.2520 \mathrm{e}-14$ & $4.0646 \mathrm{e}-14$ & $2.5939 \mathrm{e}-14$ & $1.7563 \mathrm{e}-13$ \\
$N^{2}$ & $2.1403 \mathrm{e}-14$ & $1.3372 \mathrm{e}-14$ & $4.0956 \mathrm{e}-14$ & $1.3455 \mathrm{e}-14$ & $1.0146 \mathrm{e}-13$ \\
\hline
\end{tabular}

Table 2: Computation of the integral in (56) for $x_{\ell}$ in (54) with grading factor $\alpha=1.1$. We have underlined in each column the first number, where $N_{Q} \geq N$ is satisfied (cf. Corollary 12; note that $m=O(N)$ holds in the considered case). The working precision in our MATLAB implementation is about $10^{-16}$. For this value of $\alpha$, the error is compared to a reference solution which was computed by the same method with $\max \left\{3 \cdot 10^{5}, 2 N^{2}\right\}$ quadrature points. 
Remark 24 (Application to GCQ) For the generalized convolution quadrature method, our new contour quadrature can be efficiently employed to approximate the arising contour integrals. The error analysis in [16] shows that the target accuracy $\varepsilon$ for the contour quadrature approximation has to depend on the discretization parameter $N$-for the details we refer to.[16, Theorem 11, Corollary 12]. The numerical experiments in [16] show that the choice $N_{Q}=N \log N$ preserves the overall convergence rates of the (theoretical) GCQ with exact integration.

Experiment 2: Robustness towards Uniform Time Steps We have shown numerically that our new contour quadrature allows to approximate efficiently the integral in (56) for strongly graded meshes. In the following experiment we have applied our method to the case of a very mildly graded mesh (close to uniform time stepping) to study the robustness of the quadrature method with respect to the grading factor, i.e., $\alpha$ close to 1 .

Table 2 clearly indicates that the performance of our quadrature is robust (becomes even better) as the mesh becomes close to a uniform mesh, i.e., the nodes $x_{\ell}$ in (55) cluster around a single point. This property avoids that different quadrature strategies have to be implemented depending on the strength of nonuniformity of the nodal points in the divided differences.

Experiment 3: Comparison with Standard Parametrization of the Circle and Direct Evaluation of Divided Differences Finally, our last example illustrates the dramatic improvement of our new contour quadrature in comparison to the simple parameterization $M(1+\exp (\mathrm{i} \theta)), \theta \in[0,2 \pi)$ of the circle $\mathcal{C}_{M}$ in (56) for the quadratically graded mesh. In Figure 2 we show the results for $\alpha=2$ and different values of $N$.

The evaluation of Newton's table for computing the high order divided differences in our experiment leads, in the case $\alpha=2$, to huge errors for $N \geq 64$ and even NaN approximations for $N \geq 256$. 

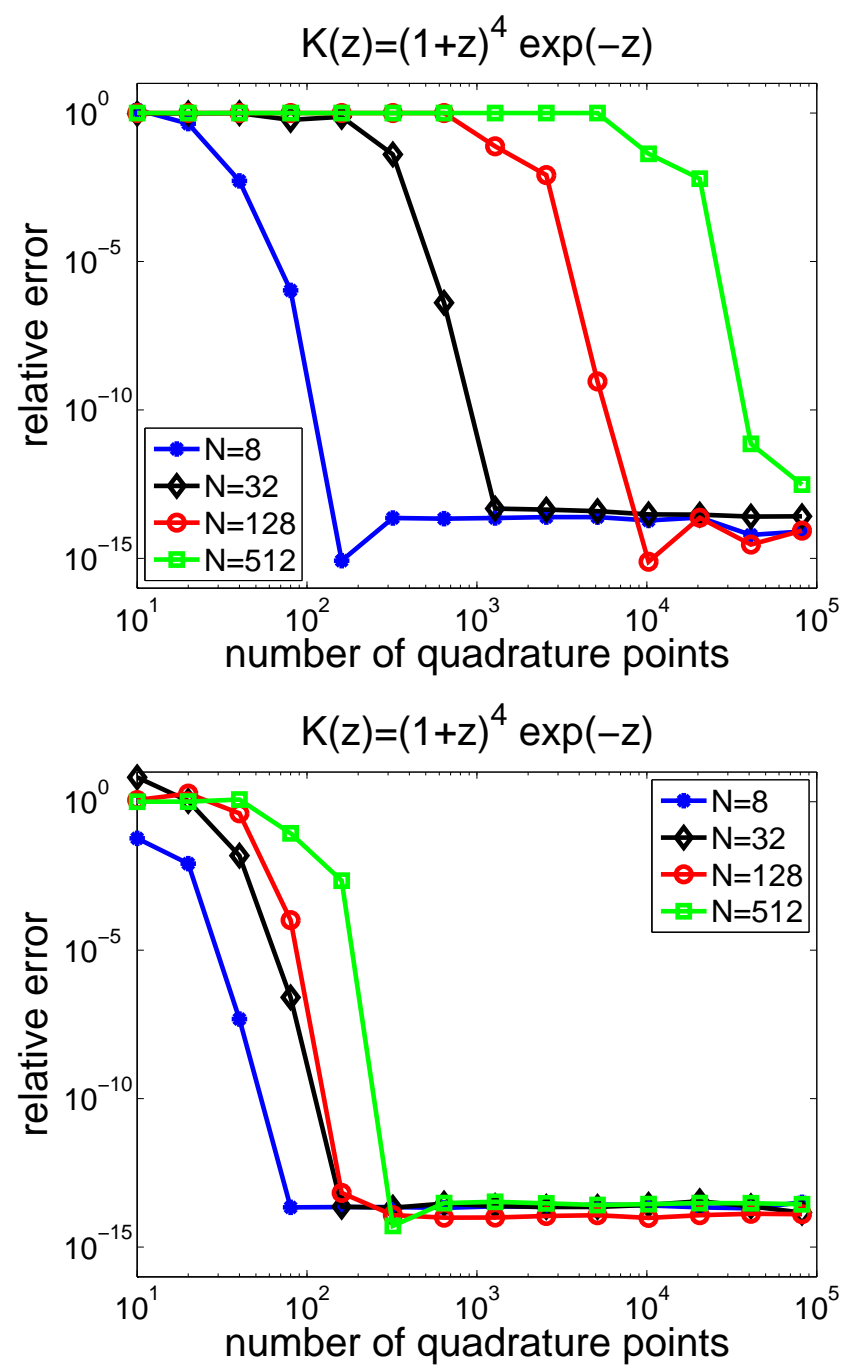

Figure 2: Convergence rates for different parameterizations of $\mathcal{C}_{M}$. Top: With the simple parametrization $M(1+\exp (\mathrm{i} \theta)), \theta \in[0,2 \pi)$. Bottom: With the parametrization in Definition 5 . 


\section{References}

[1] M. Abramowitz and I. A. Stegun. Handbook of Mathematical Functions. Applied Mathematics Series 55. National Bureau of Standards, U.S. Department of Commerce, 1972.

[2] A. Bamberger and T. H. Duong. Formulation Variationnelle Espace-Temps pur le Calcul par Potientiel Retardé de la Diffraction d'une Onde Acoustique. Math. Meth. in the Appl. Sci., 8:405-435, 1986.

[3] L. Banjai and S. Sauter. Rapid solution of the wave equation in unbounded domains. SIAM Journal on Numerical Analysis, 47:227-249, 2008.

[4] L. Banjai and M. Schanz. Wave Propagation Problems treated with Convolution Quadrature and BEM. In In Fast Boundary Element Methods in Engineering and Industrial Applications, Edited by U. Langer, M. Schanz, O. Steinbach, W.L. Wendland. Vol. 63, Lecture Notes in Applied and Computational Mechanics, pages 145-187, Chap. 5. Springer, Heidelberg, 2012.

[5] M. Caliari. Accurate evaluation of divided differences for polynomial interpolation of exponential propagators. Computing, 80(2):189-201, 2007.

[6] P. J. Davis. On the numerical integration of periodic analytic functions. In On numerical approximation. Proceedings of a Symposium, Madison, April 21-23, 1958, Edited by R. E. Langer. Publication no. 1 of the Mathematics Research Center, U.S. Army, the University of Wisconsin, pages 45-59. The University of Wisconsin Press, Madison, 1959.

[7] T. A. Driscoll. The Schwarz-Christoffel toolbox. available online at http://www.math. udel.edu/ ${ }^{\sim}$ driscoll/software/SC/.

[8] T. A. Driscoll. Algorithm 843: Improvements to the Schwarz-Christoffel toolbox for matlab. ACM Trans. Math. Software, 31:239-251, 2005.

[9] Ö. Eğecioğlu, E. Gallopoulos, and Ç. K. Koç. Fast computation of divided differences and parallel Hermite interpolation. J. Complexity, 5(4):417-437, 1989 .

[10] M. Friedman and R. Shaw. Diffraction of pulses by cylindrical obstacles of arbitrary cross section. J. Appl. Mech, 29:40-46, 1962.

[11] I. S. Gradshteyn and I. Ryzhik. Table of Integrals, Series, and Products. Academic Press, New York, London, 1965.

[12] W. Hackbusch, W. Kress, and S. Sauter. Sparse convolution quadrature for time domain boundary integral formulations of the wave equation by cutoff and panel-clustering. In M. Schanz and O. Steinbach, editors, Boundary Element Analysis, pages 113-134. Springer, 2007. 
[13] N. Hale, N. J. Higham, and L. N. Trefethen. Computing $\mathbf{A}^{\alpha}, \log (\mathbf{A})$, and related matrix functions by contour integrals. SIAM J. Numer. Anal., 46(5):2505-2523, 2008.

[14] R. Kress. Zur numerischen Integration periodischer Funktionen nach der Rechteckregel. Numer. Math., 20:87-92, 1972/73.

[15] M. Lopez-Fernandez and S. A. Sauter. A Generalized Convolution Quadrature with Variable Time Stepping. Technical Report 17-2011, Institut für Mathematik, Univ. Zürich, 2011.

[16] M. Lopez-Fernandez and S. A. Sauter. A Generalized Convolution Quadrature with Variable Time Stepping. Part II: Algorithms and Numerical Results. Technical Report 09-2012, Institut für Mathematik, Univ. Zürich, 2012 .

[17] C. Lubich. Convolution Quadrature and Discretized Operational Calculus I. Numerische Mathematik, 52:129-145, 1988.

[18] C. Lubich. Convolution Quadrature and Discretized Operational Calculus II. Numerische Mathematik, 52:413-425, 1988.

[19] A. McCurdy, K. C. Ng, and B. N. Parlett. Accurate computation of divided differences of the exponential function. Math. Comp., 43(168):501-528, 1984.

[20] K. Schiefermayr. Inequalities for the Jacobian Elliptic Functions with Complex Modulus. J. Math. Inequ., 6:91-94, 2012.

[21] A. Smoktunowicz, I. Wróbel, and P. Kosowski. A new efficient algorithm for polynomial interpolation. Computing, 79(1):33-52, 2007.

[22] J. L. Walsh. Interpolation and Approximation by Rational Functions in the Complex Domain. American Mathematical Society, New York City, 1935. 